\title{
Resultados de las técnicas blanqueadoras mixta e inmediata para el blanqueamiento de dientes tratados endodónticamente - reportes de casos
}

\author{
Results of mixed and immediate bleaching techniques for \\ bleaching endodontically treated teeth-case reports \\ O resultado das técnicas clareadoras mista e imediata para o clareamento \\ de dentes tratados endodonticamente - relatos de casos \\ Ana Claudia Dall Evedove Lopes ${ }^{1}$ (DD) 0000-0002-4495-1188 \\ José Francisco Mateo-Castillo ${ }^{1}$ (iD) 0000-0003-3727-6978 \\ Lucimara Teixeira das Neves² (iD) 0000-0003-4137-0334 \\ Lidiane de Castro Pinto ${ }^{1}$ (D) 0000-0001-9764-0327
}

\section{Resumen}

Introducción: El oscurecimiento de un diente anterior interfiere negativamente en el aspecto de la sonrisa, y varias son las causas que pueden ser responsables por este oscurecimiento. Objetivo: Describir las técnicas de blanqueamiento mixto e inmediato a través del reporte de dos casos clínicos.

Caso 1: Individuo de sexo masculino, con Síndrome de Treacher Collins, se quejó sobre alteración cromática del diente 33, verificada mediante examen clínico, radiográficamente presencia de tratamiento endodóntico satisfactorio. Por lo que fue planeado el blanqueamiento interno mediante técnica mixta.

Caso 2: Individuo de sexo masculino, con Síndrome de Apert reportó cambio cromático en el diente 22, observado en el examen clínico, radiográficamente presentando tratamiento endodóntico insatisfactorio. Se realizó retratamiento endodóntico y a los 6 meses se realizó blanqueamiento interno mediante técnica inmediata.

Conclusión: El blanqueamiento dental ejecutado con las técnicas mixtas e inmediatas, devuelve la armonía de la sonrisa, recuperando el color ideal y elevando la autoestima a los pacientes.

Palabras clave: Diente no vital, agentes blanqueadores, endodoncia.

Hospital de Reabilitação de Anomalias Craniofaciais da Universidade de São Paulo, (HRAC/USP), Bauru, SP, Brasil. lidianep@usp.br

Departamento Ciências Biológicas, Faculdade de Odontologia de Bauru, Universidade de São Paulo (FOB/USP); Orientadora de

Pós-graduação, Hospital de Reabilitação de Anomalias Craniofaciais da Universidade de São Paulo, (HRAC/USP), Bauru, SP, Brasil.

Fecha de recibido: 14/2/2020 - Fecha de aceptado: 28/1/2021 


\section{Abstract}

Introduction: The darkening of a single anterior tooth negatively affects the smile's appearance, and several factors may cause this darkening.

Objective: To describe the mixed and immediate bleaching techniques by reporting two clinical cases.

Case 1: A male individual with Treacher Collins Syndrome. He complained of chromatic alteration in tooth 33 , which was verified on clinical examination. X-ray imaging showed satisfactory endodontic treatment. Internal bleaching was performed with the mixed technique.

Case 2: A male individual with Apert Syndrome reported chromatic alteration in tooth 22 , observed on clinical examination. X-ray imaging showed unsatisfactory endodontic treatment. Endodontic retreatment was performed. Six months later, internal whitening was performed immediately.

Conclusion: The use of mixed and immediate whitening techniques restores the smile's harmony, the tooth's ideal color, and patients' self-esteem.

Keywords: Non-vital tooth, bleaching agents, endodontics.

\section{Introducción}

El blanqueamiento dental es el intento más conservador de restablecer el color normal de los dientes y presenta ventajas indiscutibles tales como: evitar el desgaste de la estructura dentaria en comparación con otros procedimientos, obtener resultados estéticos satisfactorios comprobados a largo plazo, además de ser eficiente, relativamente sencillo y barato cuando comparado al tratamiento protésico ${ }^{(1-5)}$. $\mathrm{Y}$ en casos de fracaso, no impide que terapias adicionales más

\section{Resumo}

Introduçáo: $\mathrm{O}$ escurecimento de um dente anterior interfere negativamente na aparência do sorriso, e várias são as causas que podem ser responsáveis por esse escurecimento.

Objetivo: Descrever as técnicas clareadoras mista e imediata através do relato de dois casos clínicos.

Caso 1: Indivíduo do gênero masculino com Síndrome de Treacher Collins, queixou-se de alteração cromática no dente 33, constatado no exame clínico, radiograficamente apresentando tratamento endodôntico satisfatório. O clareamento interno foi planejado e realizado pela técnica mista.

Caso 2: Indivíduo do gênero masculino, com Síndrome de Apert, relatou alteração cromática no dente 22 , constatado ao exame clínico, apresentando tratamento endodôntico insatisfatório. Foi realizada a reintervenção endodôntica e após 6 meses, foi realizado clareamento interno pela técnica imediata.

Conclusão: $\mathrm{O}$ uso das técnicas clareadoras mista e imediata, resulta na devolução da harmonia do sorriso, recuperando a coloração ideal e devolvendo a autoestima aos pacientes.

Palavras-chave: Dente não vital, agentes clareadores, endodontia.

invasivas sean realizadas, como carillas o coronas totales ${ }^{(6)}$.

Factores como trauma, calcificaciones pulpares, técnicas terapéuticas inadecuadas y fallas en la realización del tratamiento endodóntico (materiales obturadores como conos de gutapercha y cementos endodónticos) cuando dejados en el interior de la cámara pulpar, luego de finalizar el tratamiento endodóntico pueden conllevar a cambios cromáticos ${ }^{(5,7-10)}$.

Los primeros reportes en la literatura acerca del uso de agentes blanqueadores datan de 1860 , 
y el uso de diferentes sustancias, dentro de las cuales figuran: cloruro de calcio, cloro, cloruro de aluminio, ácido oxálico, dióxido de azufre, hipoclorito de sodio, entre otros ${ }^{(1)}$. El mercado ofrece agentes blanqueadores a base de peróxido de hidrógeno de $30 \%$ a $35 \%$, que puede ser utilizado separadamente o asociado al perborato de sodio ${ }^{(3,5,11)}$.

Una de las mayores preocupaciones en relación al blanqueamiento dental en dientes tratados endodónticamente es la posible aparición de reabsorción cervical externa ${ }^{(10,12)}$. Además de esta, otros posibles efectos indeseables son: reducción de la resistencia a la fractura y reducción de la microdureza dentinária en relación con la sobreexposición dentaria a estas sustancias en elevadas concentraciones y/o por largos periodos $^{(13)}$.

El presente trabajo presenta el reporte de dos casos clínicos describiendo las técnicas utilizadas para el blanqueamiento dental frente al oscurecimiento de dientes tratados endodónticamente en individuos regularmente matriculados en el Hospital de Reabilitação de Anomalias Craniofaciais da Universidade de São Paulo visto las exigencias estéticas de los pacientes.

\section{Antecedentes}

La presencia de elementos dentarios oscurecidos rompe la armonía de color de la sonrisa, lo que lleva a los pacientes a las terapias que devuelvan el color natural a dichos dientes. En los casos de dientes tratados endodónticamente y con cambio cromático, los tratamientos son diversos, sin embargo, el blanqueamiento dental se presenta como primera opción ${ }^{(6)}$.

La indicación del blanqueamiento dental en dientes no vitales debe ser criteriosa, evaluandose: presencia de restauraciones extensas o remanente insuficiente, líneas de fractura del esmalte, oscurecimiento por tetraciclina, calidad del tratamiento endodóntico, presencia de lesiones periapicales, reabsorciones externas e internas, causa y tiempo del cambio cromático y grado de oscurecimiento ${ }^{(7,9,14)}$.

Dentro de las intervenciones odontológicas, la extirpación de la pulpa provoca la hemorragia en la dentina, donde la sangre penetra en los túbulos dentinários y después se descompone; si el tejido pulpar no es completamente removido durante el tratamiento endodóntico, este puede llevar a la decoloración. Cuando el hipoclorito de sodio y la clorhexidina se mezclan se producen precipitados marrones-rojizos, que pueden causar cambio cromático, por lo que es necesario la neutralización con suero fisiologico o solución salina esteril entre el uso de estos irrigantes. A pesar de que el agregado de trióxido mineral (MTA) posee excelente biocompatibilidad, este material blanco o gris puede causar decoloraciones indeseables ${ }^{(8)}$.

Los agentes blanqueadores más utilizados en el blanqueamiento dental en dientes despulpados son el peróxido de hidrógeno, peróxido de carbamida y el perborato de sodio asociado con el agua destilada, suero fisiológico o al peróxido de hidrógeno a 35\%. Sin embargo, el empleo del perborato de sodio con agua destilada o suero fisiológico es más efectivo y seguro cuando comparado al peróxido de hidrógeno, considerado cáustico $(5,15,16)$.

El blanqueamiento para ser un procedimiento seguro, algunas condiciones deben ser respetadas: aislamiento absoluto correcto y completo de los tejidos blandos (gingivales, labios, mejillas, para protegerlos de posibles quemaduras $(17-20)$

Una de las mayores preocupaciones en relación al blanqueamiento dental en dientes tratados endodónticamente es el ph y la alta permeabilidad de los agentes blanqueadores. Cuando estos alcanzan los tejidos periodontales, a través de los túbulos dentinários, pueden generar alteración en la región de la unión amelocementária. Esto puede causar una reacción inflamatoria generada por la desnaturalización de la dentina; dicha dentina puede ser considerada un tejido diferente, siendo reconocido como un cuerpo 
extraño, consecuentemente pudiendo producir la reabsorción conocida como reabsorción cervical externa $^{(1,12,14,19,21)}$. Esta reabsorción es asintomática y es generalmente diagnosticada mediante radiografía de rutina o también mediante el examen clínico que revela hinchazón de la papila y respuesta positiva durante la percusión ${ }^{(22)}$.

Por lo tanto, es fundamental la confección de una barrera en la región cervical del diente, insertada en el nivel del límite amelocementário, entre el material obturador (gutapercha) y el espacio de la cámara pulpar, impidiendo la penetración de bacterias y previniendo la extrusión del agente blanqueador hacia el ápice o al periodonto, que podrían causar la reabsorción cervical. El cemento de ionómero de vidrio es el material más utilizado en la confección de ese tampón por poseer también un mejor costobeneficio, entre otras ventajas ${ }^{(6,14,19)}$.

Varias técnicas de blanqueamiento dental son descritas en la literatura y pueden ser clasificadas en dos grupos: de acuerdo con el local (interno o externo) o de acuerdo con el tiempo de permanencia del agente blanqueador (inmediata, mediata y mixta ${ }^{(15)}$. La gran diferencia entre estas técnicas es el método que los compuestos químicos blanqueadores liberan el oxígeno activo. En la técnica mediata o walking bleach, el agente blanqueador es colocado en el interior de la cámara pulpar y permanece por tres a siete días, siendo necesario su cambio hasta la obtención del color deseado, no sobrepasando cuatro sesiones. Esta técnica se considera la de menor riesgo de causar reabsorción cervical externa. Ya en la técnica inmediata, el agente blanqueador es aplicado en la cámara pulpar y en la superficie externa del diente y dejado por un periodo aproximado de 45 minutos. Los agentes blanqueadores son agentes oxidantes fuertes, reaccionan con los compuestos de pigmento formados por macromoléculas de carbono. A través de la oxidación, estas moléculas se convierten en compuestos de menor peso molecular (dióxido de carbono y agua) y, por lo tanto, los pigmentos se difunden a través de los tejidos mineralizados, con una acción aclarante

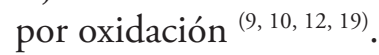

Después de lograr el efecto blanqueador deseado, el diente debe recibir una medicación con hidróxido de calcio, previamente a la restauración definitiva, por un periodo de 7-14 días con el objetivo de alcalinizar el medio y esperar el tiempo de liberación del oxígeno residual. Dicho tiempo es necesario para promover un sellado eficaz y evitar microfiltración, ya que esos residuos pueden inhibir la polimerización de los materiales adhesivos $(5,10,14,15,19)$.

A continuación, se presentan dos casos clínicos, consideraciones y resultados obtenidos.

Reporte de Caso Clínico 1: individuo de sexo masculino, 23 ańos, con Síndrome de Treacher Collins registrado en el Hospital de Reabilitação de Anomalias Craniofaciais da Universidade de São Paulo (HRAC/USP), reportó cambio cromático en el canino inferior izquierdo (33) (Fig. 1, A), constatado en el examen clínico, así como también ausencia de sintomatología dolorosa. Se observó en el examen radiográfico tratamiento endodóntico satisfactorio (Fig. 1, B). Se procedió a la ejecución del blanqueamiento dental interno mediante la técnica mixta, fue seleccionado el color usando la escala VITA (Wilcos do Brasil, Petrópolis RJ, Brasil), (color A4), y foto intrabucal (Fig. 1, C), aislamiento absoluto (Fig. 1, D), seguido de acceso a la cámara pulpar usando la fresa diamantada no 1012 (KG Sorensen Ind. Com. Ltda, Barueri, Brasil), desobturación endodóntica de aproximadamente $3 \mathrm{~mm}$ de profundidad del material obturador con condensadores no 2 calentados en la entrada del conducto radicular, limpieza de la cavidad y confección del tampón cervical (Fig. E) con cemento ionómero de vidrio (FGM, Joinville, SC, Brasil). Luego se realizó el ataque ácido de la cámara pulpar con ácido fosfórico 37\% (Biodinâmica, Ibiporá, PR, Brasil) durante 30 segundos, removido lavando con agua abundante por 60 segundos y seca- 
do con chorro de aire. Dicho paso es realizado para eliminar el "smear layer", abrir los túbulos dentinarios y, así aumentar la permeabilidad de la dentina al agente blanqueador. El gel peróxido de hidrógeno 35\% (Whiteness HP, FGM, Joinville, SC, Brasil) se manipuló siguiendo las orientaciones del fabricante y colocado en el interior de la cámara pulpar y en la cara vestibular con el auxilio de un microbrush tamaño medio (KG Sorensen Ind. Com. Ltda, Barueri, Brasil) por 45 minutos (Fig. 1, F y G); el gel se removió con agua durante 1 minuto y luego se manipuló una pasta compuesta de perborato de sodio granular (Pharmácia Specífica, Bauru, SP, Brasil) con suero fisiológico estéril en un vaso Dappen estéril, y con el auxilio de una espátula de inserción la pasta se colocó en el interior de la cámara pulpar y acomodada uniformemente (Fig. 1, H), el diente se selló con cemento ionómero de vidrio (FGM, Joinville, SC, Brasil). $\mathrm{Al}$ pasarsiete días, se observó resultado satisfactorio (Fig. 1, I y J) al compararse con el aspecto del inicio del blanqueamiento dental. El diente recibió el medicación con pasta de hidróxido de calcio Calen (sswhite Duflex, São Cristovão, RJ, Brasil) y la restauración provisional con cemento de ionómero de vidrio (FGM, Joinville, SC, Brasil). Paciente se quejó de la coloración amarillenta de los demás dientes y entonces, fue referido para el blanqueamiento externo en el sector de Odontología Estética del HRAC/USP y restauración definitiva.

Fig. 1: Secuencia clínica y radiografía del caso clínico 1
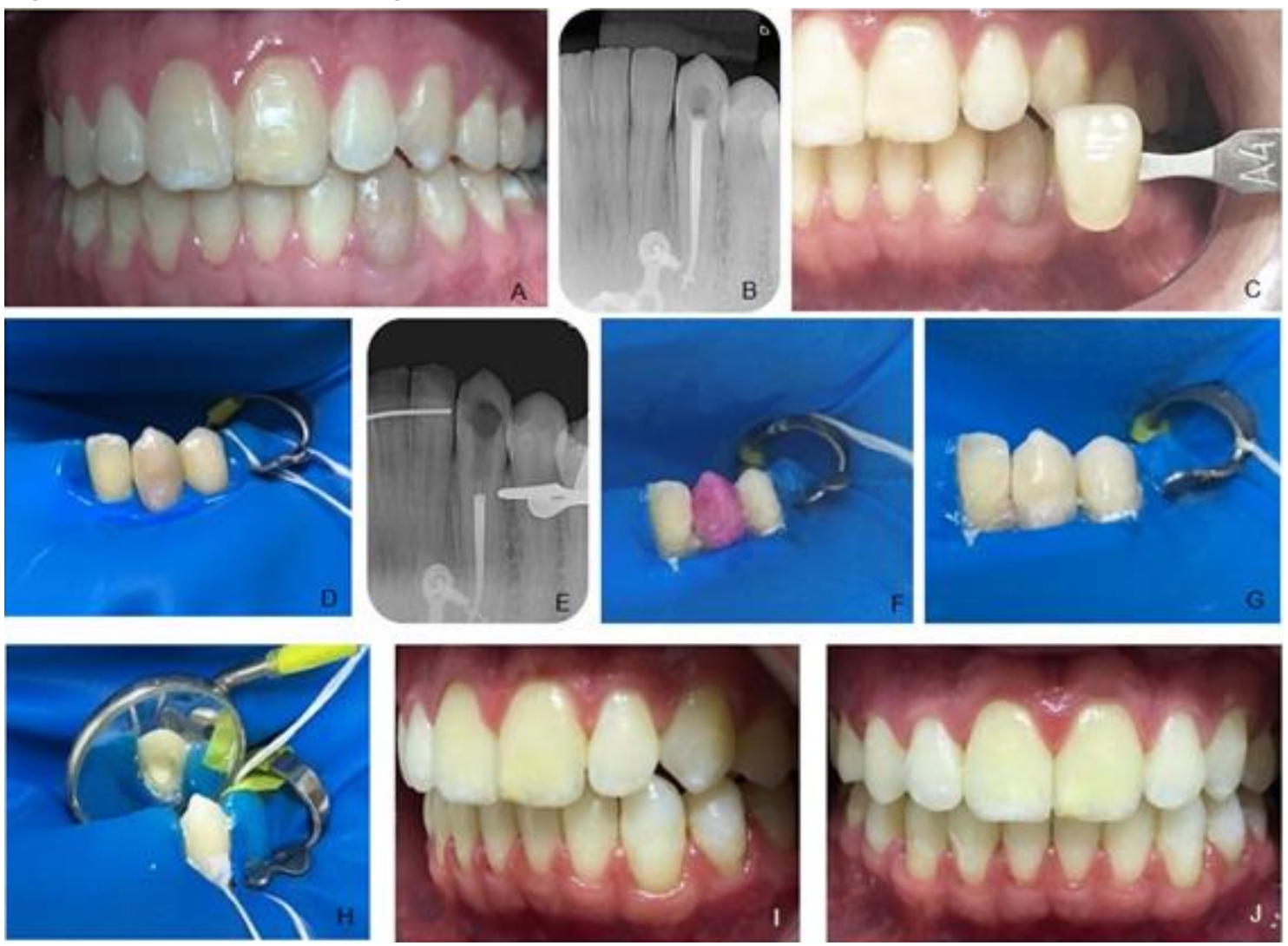
Reporte de Caso Clínico 2: individuo de sexo masculino, 30 años, con Síndrome de Apert, registrado en el HRAC/USP; reportó cambio de color en el diente superior izquierdo (22) (Fig. 2, A y B), observado en el examen clínico, así como también ausencia de sintomatología dolorosa. En la radiografia se observó tratamiento endodóntico insatisfactorio (Fig. 2, C). Se realizó el retratamiento endodóntico (Fig. 2, D y E) con necesidad de confección de un tampón apical con MTA (Fig. 2, F) (Angelus Ind. De Produtos Odontológicos S/A, Londrina, PR, Brasil). Transcurridos 6 meses de haber finalizado el retratamiento; fue iniciado el blanqueamiento dental interno mediante la técnica inmediata: el registro del color fue solo la realización de la foto intrabucal (Fig. 2, B), ya que el color del diente no se identificó en la escala VITA (Wilcos do Brasil, Petrópolis, RJ, Brasil) por ser más oscura. Se colocó entonces el aislamiento absoluto, seguido del acceso a la cámara pulpar mediante el uso de la fresa diamantada no 1012 (KG Sorensen Ind. Com. Ltda, Barueri, Brasil), desobturación endodóntica con condensadores no 2 entrada del conducto radicular, limpieza de la cavidad y confección del tampón cervical con resina fotopolimerizable surefil SDR flow (Dentsply Ind. Com. Ltda, Petrópolis, RJ, Brasil) (Fig. 2, G). Luego se realizó el ataque ácido de la cámara pulpar con ácido fosfórico 37\% (Biodinâmica, Ibiporã, PR, Brasil) durante 30 segundos y se removió con lavado abundante con agua por 60 segundos y secado con chorro de aire. El gel peróxido de hidrógeno
35\% (Whiteness HP, FGM, Joinville, SC, Brasil) se manipuló siguiendo las instrucciones del fabricante y colocado en el interior de la cámara pulpar y en la cara vestibular (Fig. 2, H y I) con el auxilio de un microbush tamaño medio (KG Sorensen Ind. Com. Ltda, Barueri, Brasil) con una aplicación de 45 minutos, el gel se removió después del lavado con agua durante $1 \mathrm{mi}$ nuto, se realizó una segunda aplicación por 25 minutos y se selló con resina surefil SDR flow (Dentsply Ind. Com. Ltda, Petrópolis, RJ, Brasil). En la segunda sesión se realizaron de igual forma el aislamiento absoluto, barrera cervical con Top Dam (FGM, Joinville, SC, Brasil), ataque ácido, lavado y secado, el gel peróxido de hidrógeno 35\% (Whiteness HP, FGM, Joinville, SC, Brasil) se manipuló siguiendo las orientaciones del fabricante e insertado en el interior de la cámara pulpar y en la cara vestibular con el auxilio de un microbush tamaño medio (KG Sorensen Ind. Com. Ltda, Barueri, Brasil) realizándose la primera aplicación por 15 minutos, luego de removerse el gel, la segunda aplicación se realizó por igual periodo, solo externamente limitada a la región cervical. Se logró resultado satisfactorio (Fig. 2, J y k) al compararse con el aspecto del inicio del blanqueamiento y restauración provisional con resina surefil SDR flow (Dentsply Ind. Com. Ltda, Petrópolis, RJ, Brasil). En el regreso, el paciente fue referido al sector de Odontología Estética donde fue realizada la restauración definitiva. 
Fig. 2: Secuencia clínica y radiográfica del caso clínico 2

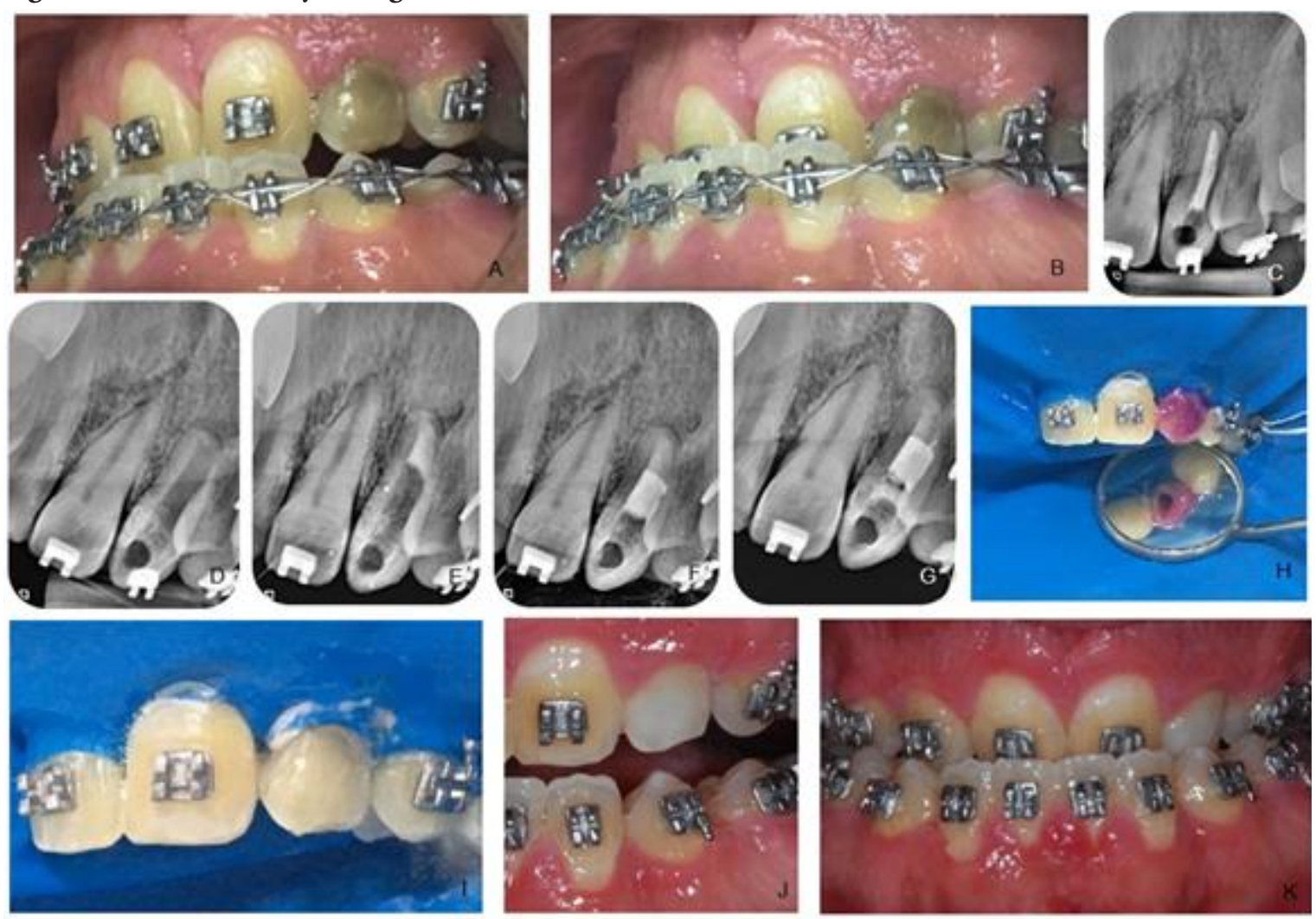

\section{Discusión}

Uno de los principales motivos de la insatisfacción estética es el cambio cromático de uno o más dientes, llevando a la búsqueda por un tratamiento que restablezca las características naturales y la armonía de la sonrisa ${ }^{(19,23)}$. Sin embargo, compete al profesional el diagnóstico y la etiología del cambio cromático para la planificación clínica, selección del material y éxito clínico del tratamiento blanqueador ${ }^{(24)}$. La presencia de diferentes materiales a menudo empleados en la obturación endodóntica en la cámara pulpar promueve considerable descoloración, siendo más intensa en el tercio cervical. Eso ocurre por el corte indebido de la obturación que debe ser realizado a nivel igual o debajo de la línea amelocementaria ${ }^{(6,11)}$, siendo esta la causa principal del os- curecimiento dental de los casos clínicos aquí descritos.

El blanqueamiento de dientes no vitales está contraindicado en situaciones de dientes con superficie vestibular ampliamente restaurada o cariada, presencia de grietas dentales, restauraciones deficientes y tratamiento endodóntico insatisfactorios. Dientes intensamente oscurecidos por muchos años poseen pequeño chance de éxito de reversibilidad de color, siendo necesaria, en algunas situaciones, la proposición de alternativas protéticas o restauradoras ${ }^{(13)}$. Por lo tanto, después del análisis de las condiciones clínicas, en el caso 1 las condiciones eran favorables para el inicio del blanqueamiento sin cualquier intervención endodóntica ya en el caso 2 se indicó el retratamiento endodóntico y, después, el blanqueamiento interno. 
El peróxido de hidrógeno $\left(\mathrm{H}_{2} \mathrm{O}_{2}\right)$ es el principal agente blanqueador empleado. Es un radical libre, térmicamente inestable, de bajo peso molecular, que penetra en el esmalte y en la dentina a través de la difusión y alcanza el periodonto siendo citotóxico ${ }^{(6,7,25)}$. Esa penetración del peróxido de hidrógeno fue significativamente más alta en dientes donde había defectos en el cemento y/o gap entre cemento y el esmalte en la unión cemento-esmalte ${ }^{(1,14)}$. Para prevenir casos de reabsorción cervical externa, que pueden ser causadas por el blanqueamiento interno de dientes no vitales, se debe evitar el uso de calor (técnica termocatalítica) y es esencial hacer un correcto sellado cervical.

El sellado cervical consiste en una barrera mecánica colocada en el nivel del límite amelocementário, entre el material obturador (gutapercha) y el espacio de la cámara pulpar, donde se colocará el agente blanqueador. Su función es impedir la penetración de bacterias o la difusión de moléculas del agente blanqueador en dirección al ápice o al periodonto, vía túbulos dentinários ${ }^{(6)}$. Ante lo expuesto, optamos por utilizar el cemento de ionómero de vidrio para la confección del tampón cervical en el caso 1 y la resina surefil SDR flow para el caso 2.

En los casos aquí descritos fueron seleccionadas técnicas diferentes, pues en el caso 1 el paciente tenía disponibilidad y posibilidad de regresar periódicamente para realizar el blanqueamiento, entonces se optó por la técnica mixta, donde además de la aplicación del gel blanqueador durante la sesión endodóntica, tanto en la cámara pulpar como externamente a la cara vestibular del diente, se aplicó una pasta con agente blanqueador internamente en la cámara pulpar y permaneció por siete días. Según la literatura es recomendado que el agente blanqueador sea cambiado o revaluado cada 7 dias, en vista que el paciente del caso clinico 2 no tenia la posibilidad de regresar para los cambios semanales se realizaron dos sesiones mediante la técnica inmediata, evitando así que el paciente retorne a su ciudad de origen con el agente blanqueador en el interior del diente.

Los agentes blanqueadores más utilizados en el tratamiento de blanqueamiento es el peróxido de hidrógeno, el peróxido de carbamida y el perborato de sodio, este último puede ser asociado con agua destilada, suero fisiológico o al peróxido de hidrógeno 35\%. Sin embargo, el empleo del perborato de sodio con agua destilada o suero fisiológico es más efectivo y seguro que con el peróxido de hidrógeno que es extremamente cáustico ${ }^{(7,26)}$. Por estas consideraciones, el caso 1, en el cual se realizó la técnica mixta, el perborato de sodio fue asociado al suero fisiológico en forma de pasta.

La efectividad de la acción de la pasta blanqueadora también depende del material utilizado en el sellado de la cavidad, que tiene la función de impedir la infiltración de fluidos en la cámara pulpar, impedir la contaminación dentinária y recoloración dental ${ }^{(24)}$. El material temporal, cemento ionómero de vidrio, se colocó para dar resistencia mecánica y al desplazamiento en consecuencia de la presión ejercida por el oxígeno liberado por la pasta blanqueadora (perborato de sodio + suero fisiológico). Se recomendó a los pacientes cuidado con relación a la consistencia del alimento ingerido y el uso del diente blanqueado en la masticación durante todo el periodo de blanqueamiento, con el fin de evitar la fractura del diente.

Los procedimientos restauradores adhesivos en el esmalte deben tener un intervalo de tiempo para su realización de 14 días de blanqueamiento dental sin alteraciones de adhesividad, en ese periodo, la cámara pulpar deberá rellenarse con una pasta de hidróxido de calcio con el fin de neutralizar el efecto de los agentes blanqueadores, por la capacidad de permear los tejidos dentales que esa pasta ofrece ${ }^{(1)}$. En los dos casos clínicos reportados utilizamos la pasta de hidróxido de calcio Calen por el periodo de 14 días y después referimos para la realización de la restauración definitiva. 
La longevidad del tratamiento blanqueador no es previsible. Mientras mayor el tiempo y el grado de oscurecimiento del diente, más sesiones clínicas serán necesarias y menor será la probabilidad de éxito en el tratamiento blanqueador. Además de la reabsorción externa, que puede ocurrir hasta 7 años después de la ejecución del tratamiento de blanqueamiento interno, ahí la importancia de la realización de control clínico y radiográfico ${ }^{(23)}$. Otra limitación es mantener el resultado a largo plazo, ya que en muchos casos puede haber recidiva del oscurecimiento, ocasionado por un sellado deficiente entre el diente y la restauración ${ }^{(23,15)}$.

\section{Conclusión}

Para los dientes tratados endodónticamente que presenten cambios cromáticos, el blanqueamiento interno presenta ventajas como; mínimo desgaste de la superficie dental, bajo costo y obtención del objetivo en poco tiempo. El uso de las técnicas mixta e inmediata, cuando bien indicadas, resultan en la devolución de la armonía de la sonrisa mediante un método sencillo, efectivo, rápido y satisfactorio. Fue posible, a través de esas técnicas, devolver la autoestima a los pacientes y la solución de los casos clínicos presentados.

\section{Referencias}

1. Boaventura JMC, Roberto AR, Lima JPM, Padovabi GC, Brisighello LC, Andrade MF. Clareamento para dentes despolpados: revisão de literatura e considerações. Rev. Odontol. Univ. Cid. São Paulo, São Paulo, 2012; 24 (2): 114-22.

2. Au U, Ma S, Lo I. Clinical efficacy of $35 \%$ carbamide peroxide and sodium perborate in intracoronal bleaching of discoloured non-vital teeth. Journal of Medicine and Biomedical, 2013, 12 (2): 96-104.

3. Murthy CS, Sudhanva M.E, Rao R., Vishwas B.V. Combined Approach for Tooth Whitening: A Case Report. Int. J Clin Dental Sci, 2011; 2(4).

4. Patil AG, Hiremath V, Kumar RS, Sheetal A, Nagaral S. Bleaching of a non-vital anterior tooth to remove the intrinsic discoloration. J Natural Science, Biology and Medicine, 2014; 5 (2).

5. Abdelkader NN. Modified Technique for Nonvital Tooth Bleaching: A Case Report. Electronic Physician, 2015; 7 (6), DOI: 10.14661/1423.

6. Hilgert LA, Néis ACC, Patrício AD, Maia HP, Andrada MAC, Edelhoff D. Clareamento de Dente Não-vital com a Técnica Inside-outside. Clín. Int. J. Braz. Dent, 2009; 5(1): $42-52$.

7. Rokaya ME, Beshr K, Hashem Mahram A, Samir Pedir S, Baroudi K. Evaluation of Extraradicular Diffusion of Hydrogen Peroxide during Intracoronal Bleaching Using Different Bleaching Agents. Int J Dent. [online] 2015; 2015: 493795. [Data de acesso: 04 de outubro de 2019]. Disponível em: https: //www.ncbi.nlm.nih.gov/pubmed/26257782

8. Souza CR, Augusto CR, Aquino EP, Alves JC, Pires RP, Venâncio GN. Reabilitação estética de dente anterior escurecido: relato de caso. Arch Health Invest [online] 2017; 6(8): 377-381. [Data de acesso 04 de outubro de 2019]. Disponível em: http: //archhealthinvestigation.emnuvens.com.br/archi/article/view/2218

9. Almohareb T. Management of Discolored Endodontically Treated Tooth Using Sodium Perborate. J Int Oral Health 2017; 9: 133-5.

10. Singh N, Chaturvedi TP, Baranwal HC, Wang CK. Management of Discolored Nonvital Tooth by Walking Bleach Technique: A Conservative Approach. J Int Clin Dent Res Organ 2020; 12: 67-71.

11. Zimmerli B, Jeger F, Lussi A. Bleaching of Nonvital Teeth A Clinically Relevant Literature Review. Schweiz Monatsschr Zahnmed. 2010; 120 (4): 306-313

12. Maciel KBL, Barbosa JS, Lins FF. Clareamento em um dente desvitalizado: relato de caso. REAS [online] 2018; (18): e83. [Data de acesso: 04 de outubro de 2019]. Disponível em: https: //acervomais. com.br/index.php/saude/article/view/83 
13. Cardoso RM, Cardoso RM, Junior PCM, Filho PFM. Clareamento interno: uma alternativa para discromia de dentes tratados endodonticamente. Odontol. Clín.-Cient. [online] 2011; 10 (2). [Data de acesso: 04 de outubro de 2019]. Disponível em: http: //revodonto.bvsalud.org/scielo.php?Script=sci_ arttext\&pid=S1677-38882011000200016

14. Attin T, Paque F, Ajam F, Lennon AM. Review of the current status of tooth whitening with the walking bleach technique. International Endodontic Journal, 36, 313-329, 2003.

15. Júnior AOS, Mateo-Castillo JF, Neves LT, Nishiyama CK, Pinto LC. Recuperação da coloração de dentes tratados endodonticamente através das técnicas clareadoras imediata e mista. Salusvita [online] 2018; 37(1): 77-91. [Data de acesso: 04 de outubro de 2019]. Disponível em: https: //secure.usc.br/ static/biblioteca/salusvita/salusvita v37 n1 2018 art 06.pdf

16. Sampaio MD, Freitas AP, Araujo RPC. Análise espectrofotométrica do clareamento dental interno / Spectrophotometric analysis of internal dental bleaching effect. RGO, Rev. Gaúch. Odontol. [online] 2010; 58(3): 363-368. [Data de acesso: 04 de outubro de 2019]. Disponível em: http: //revodonto. bvsalud.org/scielo.php?Script=sci arttext\&pid=S1981-86372010000300014\&lng=es\&nrm=iso\&tln $\mathrm{g}=\mathrm{pt}$

17. Moraru I, Tuculinã M, Bãtãiosu M, Gheorghitã, Diaconu $\mathrm{O}$. Whitening non vital teeth - a case report. Curr Health Sci J: 2012; 38(3): 132-135.

18. Dahl JE, Pallesen U. Tooth bleaching-a critical review of the biological aspects. Crit Rev Oral Biol Med, 2003; 14(4): 292-304.

19. Coelho, AS. Et al. Non-Vital Tooth Bleaching Techniques: A Systematic Review. Coatings 2020, 10(1), 61; https: //doi.org/10.3390/coatings10010061

20. Rodrigues LM, Vansan LP, Pécora JD, Marchesan MA. Permeability of different groups of maxillary teeth after 38 percent hydrogen peroxide internal bleaching. Braz. Dent. J, 2009; 20 (4): 3036.

21. Badole GP, Warhadpande MM, Bahadure RN, Badole SG. Aesthetic Rehabilitation of Discoloured Nonvital Anterior tooth with Carbamide Peroxide Bleaching: Case Series. J Clin Diagn Res. 2013; 7 (12): 3073-3076.

22. Nagaveni NB, Umashankara KV, Radhika NB, Satisha TS. Management of tooth discoloration in non-vital endodontically treated tooth - A report of 6 year follow-up. J Clin Exp Dent. 2011; 3(2): 180-183.

23. Bortolatto JF, Corsi CE, Presoto CD, Cioffi SS, Júnior OBO. Clareamento interno em dentes despolpados como alternativa a procedimentos invasivos: relato de caso. Rev. Odontol. Univ. Cid. Sáo Paulo, São Paulo, 2012; 24 (2): 142-52.

24. Kasuya AVB, Favarão IN, Souza JL, Cardoso SA, Fonseca RB, Hoeppner MG. Longevidade de clareamento em dente não vital com associação de técnicas: relato de caso. Estética 2012; 9 (2): 104-113.

25. Fernandes AM, Marques MM, Camargo SEA, Cardoso PE, Camargo HR, Valera MC. Cytotoxicity of non-vital dental bleaching agents in human gingival fibroblasts. Braz Dent. Sci 2013; 16 (1): 59-65.

26. Valera MC, Camargo CH, Carvalho CA, de Oliveira LD, Camargo SE, Rodrigues CM. Effectiveness of carbamide peroxide and sodium perborate in non-vital discolored teeth. J Appl Oral Sci. [online] 2009; 17(3): 254-61. [Data de acesso: 04 de outubro de 2019]. Disponível em: http: //www.scielo.br/ scielo.php?Script=sci arttext\&pid=S1678-77572009000300024 


\section{Nota declaración de interés:}

Los autores declaran que no tienen intereses comerciales, de propiedad o financieros que representen conflictos de interés en los productos y compañías descritas en este artículo.

\section{Nota contribución de los autores:}

1. Concepción y diseño del estudio

2. Adquisición de datos

3. Análisis de datos

4. Discusión de los resultados

5. Redacción del manuscrito

6. Aprobación de la versión final del manuscrito

ACDEL ha contribuido en: 1, 2, 3, 4, 5 y 6 .

JFMC ha contribuido en: 3, 4 y 5 .

LTN ha contribuido en: 3,4 y 5 .

LCP ha contribuido en: 2, 3, 4 y 5 .

\section{Nota de aceptación:}

Este artículo fue aprobado por la editora de la revista Mag. Dra. Vanesa Pereira-Prado. 